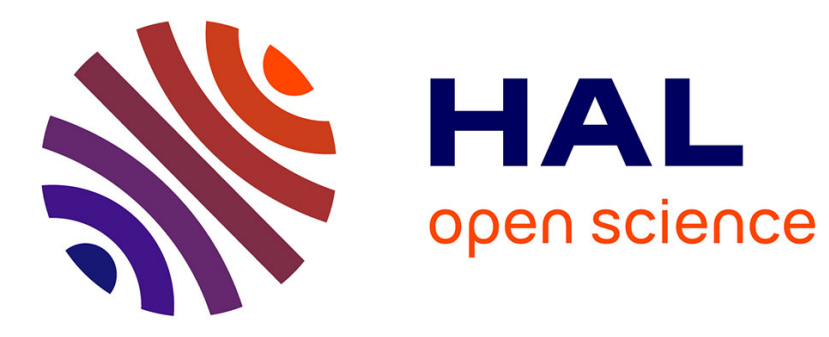

\title{
Neutron microscopy
}

A. Steyerl, W. Drexel, T. Ebisawa, E. Gutsmiedl, K.-A. Steinhauser, R. Gähler, W. Mampe, P. Ageron

\section{To cite this version:}

A. Steyerl, W. Drexel, T. Ebisawa, E. Gutsmiedl, K.-A. Steinhauser, et al.. Neutron microscopy. Revue de Physique Appliquée, 1988, 23 (2), pp.171-180. 10.1051/rphysap:01988002302017100 . jpa00245760

\section{HAL Id: jpa-00245760 https://hal.science/jpa-00245760}

Submitted on 1 Jan 1988

HAL is a multi-disciplinary open access archive for the deposit and dissemination of scientific research documents, whether they are published or not. The documents may come from teaching and research institutions in France or abroad, or from public or private research centers.
L'archive ouverte pluridisciplinaire HAL, est destinée au dépôt et à la diffusion de documents scientifiques de niveau recherche, publiés ou non, émanant des établissements d'enseignement et de recherche français ou étrangers, des laboratoires publics ou privés. 
Classification

Physics Abstracts

$07.80-29.25 \mathrm{~F}-41.80$

\title{
Neutron microscopy
}

\author{
A. Steyerl, W. Drexel ( $\left.{ }^{1}\right)$, T. Ebisawa $\left({ }^{*}\right)$, E. Gutsmiedl, K.-A. Steinhauser (**), R. Gähler, \\ W. Mampe ( ${ }^{1}$ ) and P. Ageron ( $\left.{ }^{1}\right)$
}

Fakultät für Physik, Technische Universität München, D-8046 Garching, F.R.G.

(1) Institut Laue-Langevin, F-38042 Grenoble, France

(Reçu le 28 août 1987, accepté le 19 novembre 1987)

\begin{abstract}
Résumé. - On donne les performances d'un microscope à neutrons, achromatique à deux miroirs, essayé à l'Institut Laue-Langevin au moyen de la nouvelle source à haute intensité de neutrons ultrafroids. On a observé une résolution d'environ $10 \mu \mathrm{m}$ avec des magnifications jusqu'à 240 . On présente également une analyse générale de la formation d'images par des neutrons ultrafroids au moyen de systèmes optiques avec un nombre quelconque de miroirs. Cette analyse facilite une discussion des contraintes géométriques pour des systèmes achromatiques à haut degré. On analyse en détail une classe particulière de systèmes à quatre miroirs aplanatiques et achromatiques.
\end{abstract}

\begin{abstract}
We report on the performance of the achromatic two-mirror neutron microscope at the Institut Laue-Langevin as investigated at the new strong source of ultracold neutrons. Using magnifications of up to 240 a resolution of about $10 \mu \mathrm{m}$ was observed. We also present a general analysis of UCN imaging by optical systems with an arbitrary number of mirrors. On this basis the geometrical constraints for highly achromatic systems are discussed. A specific class of aplanatic and achromatic four-mirror systems is analysed in detail.
\end{abstract}

\section{Introduction.}

Neutrons interact with matter in a different way than do light or electrons. Since the first proposal by I. M. Frank [1] various imaging systems for neutrons have been developed [1-10] allowing us to use the specific neutron contrast for microscopy. In two recent works at Garching/Grenoble [6] and at Moscow/Leningrad [9] two-mirror systems for ultracold neutrons were employed, choosing geometrical configurations where the chromatic aberrations induced by the gravitational beam curvature are compensated in lowest order.

For this purpose Herrmann et al. [4, 6] have developed a system of two mirrors arranged along a common vertical optical axis, where the neutrons pass the highest point of their flight parabola on their way from the first to the second mirror. It follows from their analysis that all confocal configurations (where the optical focal points of the two

(*) On leave from : Research Reactor Institute, Kyoto University, Kumatori-Cho, Sennan-Gun, Osaka, Japan.

(**) Present address : Siemens AG, Hofmannstr. 51, D8000 München, F.R.G. mirrors exactly coincide) are achromatic to lowest order, both with respect to image position and to magnification. First results at a magnification of 50 were reported in 1985 [6]. The first part of the present paper describes the progress made since this microscope was transferred to the new, intense Turbine Source [11] of ultracold neutrons at the High-Flux Reactor of the Institut Laue-Langevin, Grenoble.

In 1986 Arzumanov et al. showed [9] that a second class of two-mirror systems is capable of compensating the two lowest-order chromatic aberrations. The neutrons travel along a vertical flight path between the two concave mirrors (without reversal of flight direction), and the mirror separation should equal approximately one sixth of the sum of the mirror radii of curvature. This particular solution is not contained in the analysis of [6] which excludes the case where the intermediary image distance diverges. First two-dimensional images were obtained for this scheme at a magnification of 1.4 [9, 10].

In a different approach Frank and his collaborators $[12,13]$ have proposed to eliminate the gravitationally induced aberrations by a compen- 
sation of gravity for neutrons by the action of an inhomogeneous magnetic field.

In the second part of the present paper we extend UCN mirror optics to systems with an arbitrary number of mirrors arranged on top of one another, as well as to higher order chromatic aberrations under the influence of gravity. We also address the question of mirror shapes and the possibility of reducing the geometrical aberrations along with chromatism. Finally, a class of achromatic fourmirror systems is proposed which permits us to use arbitrarily large magnifications at an $f$-value of about 1.5 and a resolution of a few microns.

\section{The two-mirror confocal system.}

The microscope set-up shown in figure 1 has been described in detail in $[4,6,7]$. This instrument has been transferred to the new UCN Turbine Source [11] at the High-Flux Reactor, Grenoble, which provides a 100 times stronger beam than the previous source PN5 [14]. The UCN current amounts to about $6 \times 10^{3} \mathrm{~cm}^{-2} \mathrm{~s}^{-1}$ for UCN velocities up to $6.2 \mathrm{~m} / \mathrm{s}$, over a beam cross-section of $8 \times 15 \mathrm{~cm}^{2}$.

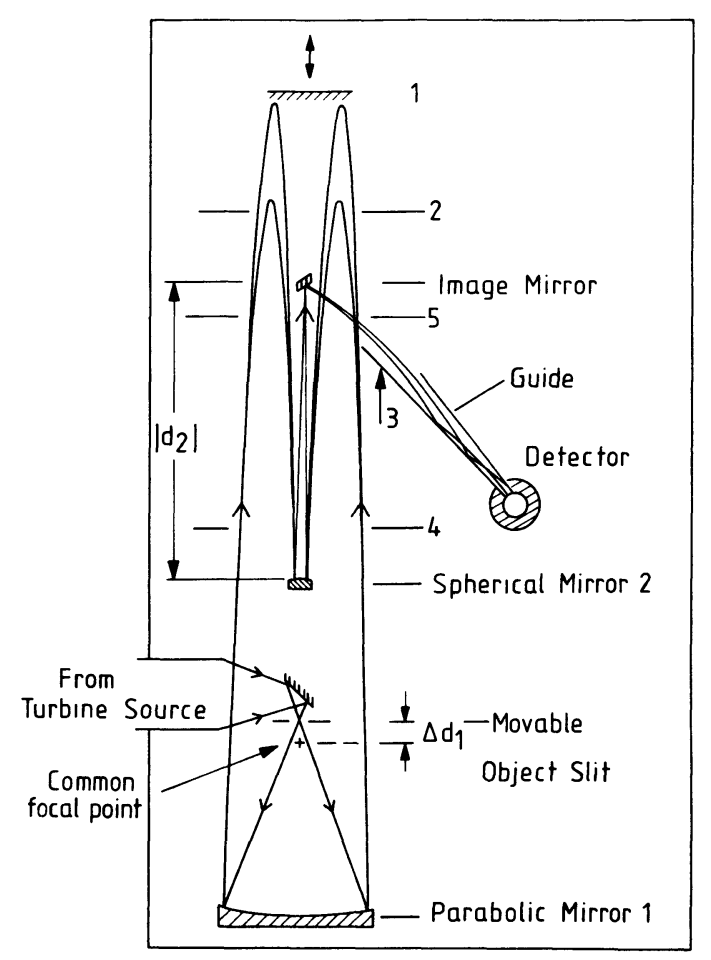

Fig. 1. - Scheme of the two-mirror microscope set-up. The neutrons pass the highest point of their flight parabola between the confocal imaging mirrors. This system is free of chromatism of second order, both in image position and in magnification. Beam tailoring and rejection of erratic neutrons are achieved by use of the beam stops 1 to 5 . The instrument is designed for a wide entrance aperture (1:1.5) and can be set to arbitrarily high magnifications. The mirrors are nickel coated (the primary mirror with $\left.{ }^{58} \mathrm{Ni}\right)$.
Leaving the first, concave-parabolic mirror of radius of curvature $\left|R_{1}\right|=585.2 \mathrm{~mm}$ and diameter $20 \mathrm{~cm}$ unchanged, the optical characteristics of this system were investigated using various radii of curvature $R_{2}$ for the second, convex-spherical mirror of diameter $4.5 \mathrm{~cm}$. Achromatism in lowest order for confocal systems of this type is established in the following form : for given values of $R_{1}, R_{2}$, and a fixed object position (slightly above the light-optical focus of the first mirror) the system selects from the wide range of primary neutron velocities (up to about $7.3 \mathrm{~m} / \mathrm{s}$, the critical velocity for total reflection at the ${ }^{58} \mathrm{Ni}$ coating on the first mirror) a band about a characteristic velocity $V_{0}$ (which we measure at the level of the first mirror) for which the chromatic aberrations $\left(\partial d_{2} / \partial v\right)_{d_{1}=\text { const. }}$ and $(\partial M / \partial v)_{d_{1}}$ vanish. $d_{1}$ and $d_{2}$ denote the object and image distance, respectively, and $M$ is the overall magnification. Figure 2 shows the optical characteristics for two different radii (and appropriate mirror separations such as to ensure confocality) for the second mirror : $\left|R_{2}\right|=1.2232 \mathrm{~m}$ and $\left|R_{2}\right|=\left|R_{1}\right|=0.5852 \mathrm{~m}$. The former value had been used in the original design [4, 6]. We observe from figure 2 that different ranges of magnification (up to arbitrarily large values) may be selected by an appropriate choice of $R_{2}$ - more generally : of $R_{2} / R_{1}$ - and that the desired value within these ranges may be set by the choice of the image distance $d_{2}$. Focussing is then accomplished by an adjustment of object position. The selection of the desired wavelength band about the design velocity $V_{0}$ is in practice achieved by beam stops as

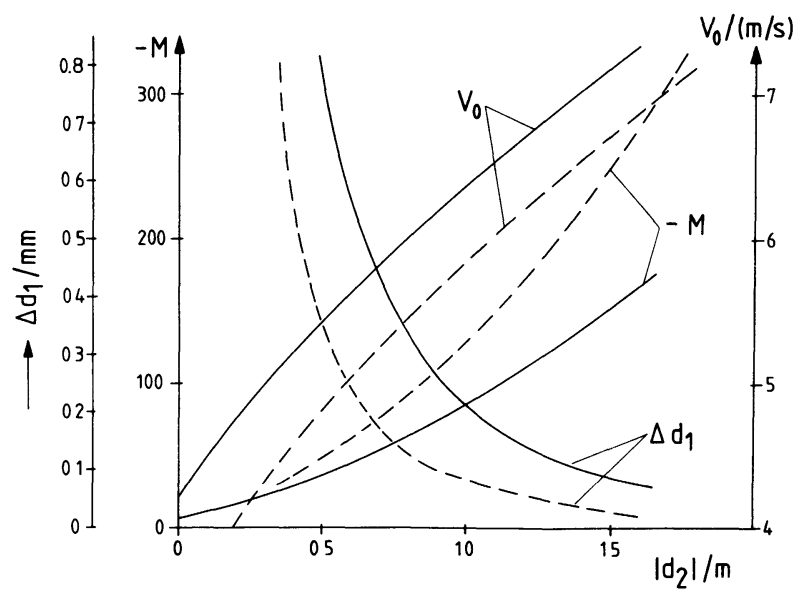

Fig. 2. - Characteristics of the confocal two-mirror microscope. For given mirror geometry $\left(R_{1}, R_{2}\right.$, and mirror separation $\left.\left|R_{1}-R_{2}\right| / 2\right)$ the magnification can be chosen within a certain range by appropriate positioning of object and image ( $\Delta d_{1}$ and $\left|d_{2}\right|$, respectively). The system then selects a certain velocity range about the stationary value $V_{0}$ which depends on the setting. The characteristics are shown for $\left|R_{1}\right|=0.5852 \mathrm{~m}$ and two values of $\left|R_{2}\right|: 1.2232 \mathrm{~m}$ (solid curves) and $0.5852 \mathrm{~m}$ (dashed curves). 
indicated in figure 1, which limit the maximum neutron ascent and serve as a protection against stray neutrons and undesired trajectories at the same time.

Results of object scans for a slit of 0.4 to $1 \mathrm{~mm}$ in width and 1.4 to $4 \mathrm{~mm}$ in length are shown in figure 3 for the following microscope settings :

a) Same setting as in reference [6]: slit $1 \times$ $4 \mathrm{~mm}^{2},\left|R_{2}\right|=1.2232 \mathrm{~m}, \Delta d_{1}=0.44 \mathrm{~mm}$ above the focal point (for optimal setting), $\left|d_{2}\right|=662.7 \mathrm{~mm}$ above the second mirror, $M=-51, V_{0}=5.73 \mathrm{~m} / \mathrm{s}$, velocity range $\Delta V_{0}={ }_{-0.25}^{+1} \mathrm{~m} / \mathrm{s}$;

b) Slit $0.4 \times 2 \mathrm{~mm}^{2}, \quad\left|R_{2}\right|=1.6079 \mathrm{~m}, \quad \Delta d_{1}=$ $0.29 \mathrm{~mm}, \quad\left|d_{2}\right|=924.2 \mathrm{~mm}, \quad M=-79, \quad V_{0}=$ $6.68( \pm 0.5) \mathrm{m} / \mathrm{s}$;

c) Slit $0.4 \times 1.4 \mathrm{~mm}^{2},\left|R_{2}\right|=585.2 \mathrm{~mm}, \Delta d_{1}=$ $0.03 \mathrm{~mm},\left|d_{2}\right|=1009.6 \mathrm{~mm}, \quad M=-243, \quad V_{0}=$ $6.68( \pm 0.5) \mathrm{m} / \mathrm{s}$.
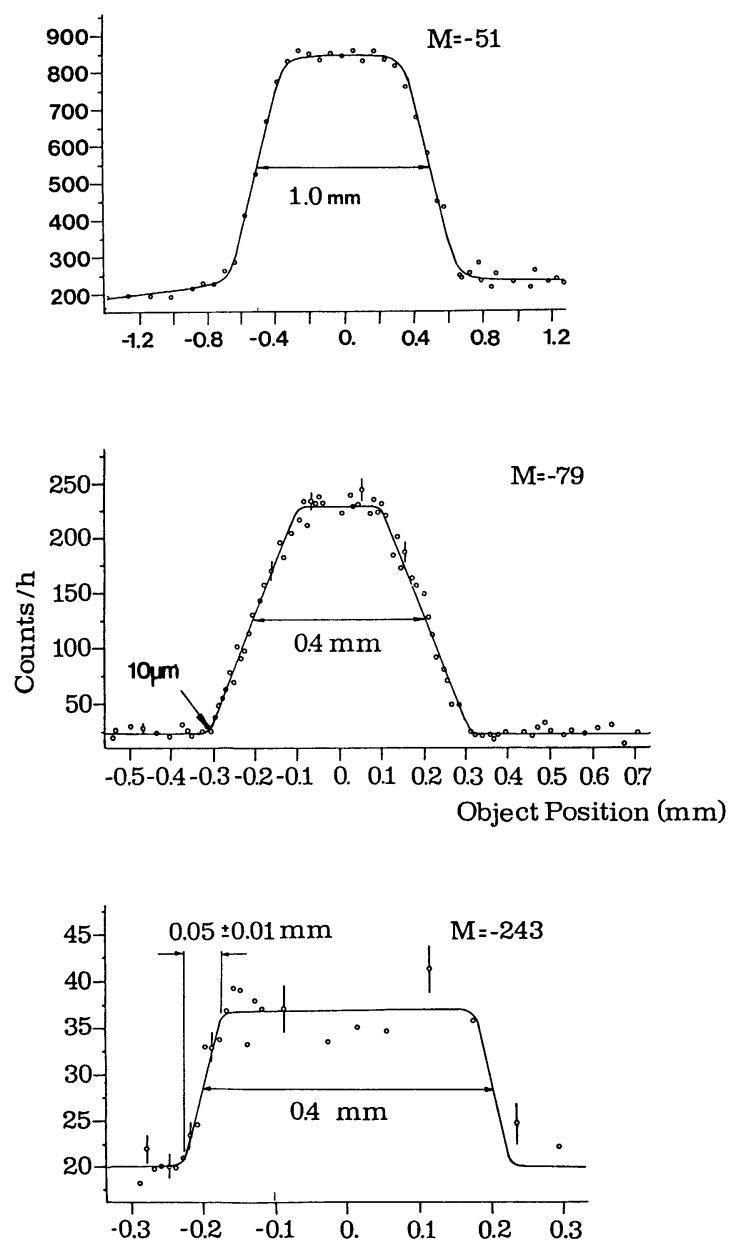

Fig. 3. - Object scans for slits of various size and for different settings of the confocal two-mirror microscope. The edge width $q /|M|$ of the observed trapezoids is determined by the effective image mirror size $q=$ $16.2 \mathrm{~mm}$ and by the magnification $M$. The aberration limit of $\sim 10 \mu \mathrm{m}$ to the resolution may be derived mainly from the corner blurring.
The optical properties of the microscope are derived from the observed image features in the following way: the edge width of the image trapezoid is determined by $q /|M|$ where $q=$ $16.2 \mathrm{~mm}$ corresponds to the image resolution. In the present design with only one image scanning element it is given by the width, projected into the horizontal plane, of the $17 \times 17 \mathrm{~mm}^{2}$ image scanning mirror which is tilted by $18^{\circ}$ to the horizontal plane. Aberrations as well as deviations from the exact focussing condition tend to reduce the edge slope and to blur the corners. This behaviour was investigated in ray-tracing computer simulations. They displayed that, except in highly defocussed conditions, the blurred edge profiles may be represented by a superposition of two hyperbolas which join with continuous slope at the edge centre and asymptotically approach the background and plateau regions, respectively. The measured magnification and resolution may thus be derived from a fit of this model to the data.

The main features observed are as follows :

a) The aberration limit to object resolution, as determined from the edge blurring, is about $10 \mu \mathrm{m}$. As noted previously in [6], the resolution of the present scheme of a microscope is limited mainly by the geometrical aberrations coma and spherical aberration. The analysis in section 4 below shows that the spherical aberrations given by $\partial^{m} A / \partial \phi^{m}$ (for $m=3,5,7, \ldots$ ) can be eliminated by the choice of an elliptical shape for the first mirror (i.e., by a slight departure from the parabola). Coma ( $\propto \partial^{3} A / \partial r \partial \phi^{2}$ ) cannot be corrected in the present scheme. It lies in the range 10 to $20 \mu \mathrm{m}$ for all settings considered, where the numerical values are based on an image field width of $r_{\max }|M|=3 \mathrm{~cm}$ in radius and on a wide entrance aperture $\left(2 \phi_{\max }=\right.$ $0.72 \mathrm{rad}$, corresponding to $f=1.5$ ). As usual, the total aberration $A$ is defined as the distance in the image plane by which a ray misses the nominal image point if it leaves a given point $r$ in the object plane at a given angle $\phi$ and speed $v_{0} r$ and $\phi$ are measured from the optical axis.

b) For the present set-up the measured image intensity corresponds to an effective object illumination of about $2 \mathrm{~s}^{-1} \mathrm{~mm}^{-2}$ (factor 125 above the corresponding value on PN5). Thus the possibility of exploiting the intrinsic resolution limit of $\sim 10 \mu \mathrm{m}$ crucially depends on the availability of very strong UCN sources as well as of two-dimensionally position-sensitive detection. Schemes for further advanced UCN sources have been developed [15], and the successful operation of a scintillation camera for UCN has been reported [9]. Work on the development of a detector for $v \cong 2 \mathrm{~m} / \mathrm{s}$, the design velocity in the image plane for the present scheme (nearly independent of microscope setting), is in progress. 
In the following we present a general analysis of UCN optics in systems of an arbitrary number $n$ of mirrors with a common, vertical optical axis. We also address the problem of optimal mirror shapes. Based on these considerations we then discuss a special set of four-mirror systems of high speed and minimized chromatic and geometrical aberrations.

\section{Neutron imaging by an $n$-mirror system $(n \geqslant 2)$.}

For a system of $n$ mirrors hit successively by a neutron on its travel from object to image we introduce a system of cartesian coordinates where $z$ points in the direction of the gravitational acceleration $\left(^{1}\right) \mathbf{g}$, and we use the following nondimensional variables: $\xi_{i}=v_{i} / V_{0}$ for the velocity at mirror $i$ $(1 \leqq i \leqq n) ; \tau_{i j}=v_{i j} / V_{0}$ - velocity at (actual or intermediary) object point $(j=1)$ or image point $(j=2)$ before and after mirror $i$, respectively; $\alpha_{i j}=\tau_{i j}-\xi_{i}=\left(g / V_{0}\right) t_{i j}$ - nondimensional flight time between mirror $i$ and the respective (intermediary) object/image positions ;

$$
\begin{aligned}
\delta_{i j} & =\alpha_{i j}\left(\xi_{i}+\alpha_{i j} / 2\right) \\
& =\left(\tau_{i j}^{2}-\xi_{i}^{2}\right) / 2=\left(g / V_{0}^{2}\right) d_{i j}
\end{aligned}
$$

- nondimensional (intermediary) object/image distance (positive/negative for positions below/above mirror $i)$;

$$
\mu_{i}=t_{i 2} / t_{i 1}=\alpha_{i 2} / \alpha_{i 1}
$$

- partial magnification for mirror $i ; \rho_{i}=g R_{i} / V_{0}^{2}$ - nondimensional mirror radius of curvature (positive/negative for focal point below/above the mirror). $s_{i}=\left(g / V_{0}^{2}\right) S_{i}$ denotes the vertical distance of mirror $i$ below the reference level (which is arbitrarily chosen as the position of mirror 1 ). The present nomenclature is not identical to that used in [6].

The connection between the successive mirror subsystems $i$ and $i+1$ via the common intermediary image/object may be expressed as

$$
\tau_{i 2}=\tau_{(i+1) 1} .
$$

The imaging equation [1] for mirror $i, 2 / R_{i}=$ $\left(1 / v_{i}\right)\left(t_{i 1}^{-1}+t_{i 2}^{-1}\right)$, reads in terms of these dimensionless quantities :

$$
\xi_{i} \sigma_{i}=\alpha_{i 1}^{-1}+\alpha_{i 2}^{-1},
$$

where $\sigma_{i}^{-1}=\rho_{i} / 2$ is the nondimensional focal length of mirror $i$. The sign conventions adopted here imply that in proceeding from subsystem $i$ to

( $\left.{ }^{1}\right)$ Gravity could be replaced by any potential varying only with $z$ like $U \sim z$, as e.g. (for neutrons) a magnetic field distribution with uniform gradient of $|\mathbf{B}|$. $i+1$ we have to choose respectively $\operatorname{sgn}\left(\xi_{i+1}\right)=$ $-\operatorname{sgn}\left(\xi_{i}\right)$ or $\operatorname{sgn}\left(\xi_{i+1}\right)=+\operatorname{sgn}\left(\xi_{i}\right)$, depending on whether the particle has or has not passed an apex of its parabolic flight path on its way between mirrors $i$ and $i+1$. Equation (2) implies useful linear relations between $\tau_{i j}$ and the partial magnification :

and

$$
\begin{aligned}
\mu_{i} & =-1-\sigma_{i} \xi_{i}^{2}+\sigma_{i} \xi_{i} \tau_{i 2} \\
\mu_{i}^{-1} & =-1-\sigma_{i} \xi_{i}^{2}+\sigma_{i} \xi_{i} \tau_{i 1} .
\end{aligned}
$$

The analysis of $n$-mirror systems may be placed on a systematic basis by use of the following recursion relations which may be obtained from (1-2) :

$$
\tau_{i 2}=\left(E_{i}+B_{i} \tau_{(i-1) 2}\right) /\left(C_{i}+D_{i} \tau_{(i-1) 2}\right),
$$

and

$$
\begin{aligned}
\left(\mu_{i} \mu_{i-1}\right)^{-1} & =g_{i-1}^{i}+h_{i-1}^{i} \mu_{i-1}^{-1} \\
\mu_{i-1} \mu_{i} & =g_{i}^{i-1}+h_{i}^{i-1} \mu_{i}
\end{aligned}
$$

or

where

$$
\begin{gathered}
E_{i}=2 \xi_{i} \gamma_{i}^{2} ; \quad B_{i}=-\left(\xi_{i}^{2}+\gamma_{i}^{2}\right) ; \\
C_{i}=-B_{i} ; \quad D_{i}=-2 \xi_{i}=-E_{i} / \gamma_{i}^{2}
\end{gathered}
$$

(apart from an arbitrary multiplying factor)

$$
\begin{aligned}
& \text { and (for } \nu=i-1 \text { or } i \text { ) } \\
& \left.\begin{array}{rl}
g_{\nu \pm 1}^{\nu \pm} & =\sigma_{\nu \pm 1} \xi_{\nu \pm 1} /\left(\sigma_{\nu} \xi_{\nu}\right)=\left(g_{\nu \pm 1}^{\nu}\right)^{-1} \\
h_{\nu \pm 1}^{\nu} & =g_{\nu \pm 1}^{\nu}-1-\sigma_{\nu \pm 1} \xi_{\nu \pm 1}\left(\xi_{\nu \pm 1}-\xi_{\nu}\right) \\
& =-h_{\nu \pm 1}^{\nu} / g_{\nu \pm 1}^{\nu}
\end{array}\right\} .
\end{aligned}
$$

These relations are valid for any neutron velocity. Designating the departure from the design value $V_{0}$ by $\gamma=v_{0} / V_{0}$ (again measured at mirror 1, i.e., $\left.v_{1}=v_{0}\right)$ we obtain $\xi_{i}= \pm\left(\gamma^{2}+2 s_{i}\right)^{1 / 2}$, with the sign specification given above. $\gamma_{i}^{2}=\left(v_{f i} / V_{0}\right)^{2}=$ $\xi_{i}^{2}+\rho_{i}$ is a measure of velocity $v_{f i}$ at the focus of mirror $i$.

The recursion relations (3-4) can be used to derive the transfer characteristics for $n$-mirror imaging in the simple form

$$
\tau_{n}=\left(E+B \tau_{1}\right) /\left(C+D \tau_{1}\right)
$$

and

or

$$
\begin{gathered}
M^{-1}=G_{1}+H_{1} \mu_{1}^{-1}=M_{1}^{-1}+X_{1} \tau_{1}, \\
M=G_{n}+H_{n} \mu_{n}=M_{n}+X_{n} \tau_{n},
\end{gathered}
$$

where $\tau_{1}=\tau_{11}$ and $\tau_{n}=\tau_{n 2}$ respectively refer to object and final image, and $M=\mu_{1} \mu_{2} \ldots \mu_{n}$ denotes the overall magnification. The transfer coefficients may be calculated by successive application of (3) and (4), or in matrix formulation : 


$$
\left.\begin{array}{l}
\left(\begin{array}{cc}
E & B \\
C & D
\end{array}\right)=\left(\begin{array}{ll}
0 & 1 \\
1 & 0
\end{array}\right)\left(\begin{array}{ll}
C_{n} & D_{n} \\
E_{n} & B_{n}
\end{array}\right) \ldots\left(\begin{array}{ll}
C_{2} & D_{2} \\
E_{2} & B_{2}
\end{array}\right)\left(\begin{array}{ll}
C_{1} & D_{1} \\
E_{1} & B_{1}
\end{array}\right) \\
\left(G_{1} ; H_{1}\right)=(0 ; 1)\left(\begin{array}{cc}
0 & 1 \\
g_{n-1}^{n} & h_{n-1}^{n}
\end{array}\right) \ldots\left(\begin{array}{cc}
0 & 1 \\
g_{2}^{3} & h_{2}^{3}
\end{array}\right)\left(\begin{array}{cc}
0 & 1 \\
g_{1}^{2} & h_{1}^{2}
\end{array}\right) \\
\left(\begin{array}{c}
G_{n} \\
H_{n}
\end{array}\right)=\left(\begin{array}{ll}
0 & g_{n}^{n-1} \\
1 & h_{n}^{n-1}
\end{array}\right) \ldots\left(\begin{array}{ll}
0 & g_{3}^{2} \\
1 & h_{3}^{2}
\end{array}\right)\left(\begin{array}{ll}
0 & g_{2}^{1} \\
1 & h_{2}^{1}
\end{array}\right)\left(\begin{array}{l}
0 \\
1
\end{array}\right)
\end{array}\right\}
$$

The quantities $M_{1}=\left[G_{1}-H_{1}\left(1+\xi_{1}^{2} \sigma_{1}\right)\right]^{-1}$ and $M_{n}=G_{n}-H_{n}\left(1+\xi_{n}^{2} \sigma_{n}\right)$ denote the magnification for the cases where respectively the object or the final image is located at the level of maximum vertical reach for the neutron velocity $v_{0}$ (i.e., for $\tau_{1}=0$, or $\tau_{n}=0$ ). Further, we have used the abbreviations $X_{1}=\xi_{1} \sigma_{1} H_{1}$ and $X_{n}=\xi_{n} \sigma_{n} H_{n}$.

The above quantities are not all independent. Using (7) and the consistency condition

$$
1=M^{-1} M=\left(M_{1}^{-1}+X_{1} \tau_{1}\right)\left(M_{n}+X_{n} \tau_{n}\right)
$$

the following relations may be established :

$$
\begin{gathered}
E=M_{1}-M_{n} ; B=-X_{1} M_{1} M_{n} ; C=X_{n} ; \\
D=X_{1} X_{n} M_{1}
\end{gathered}
$$

(again apart from an arbitrary common multiplier).

We add in passing that a further recursion relation may be set up for the quantity $\beta_{i}=$ $\left(\alpha_{i 1}^{-1}-\alpha_{i 1}^{*-1}\right)^{-1}=-\left(\alpha_{i 2}^{-1}-\alpha_{i 2}^{*-1}\right)^{-1}$. The $\beta^{\prime}$ s relate the flight times $\alpha_{i j}$ to the quantities $\alpha_{i j}^{*}=$ $(-1)^{j+1} \gamma_{i}-\xi_{i}$ (for $\left.1 \leqq i \leqq n, j=1,2\right)$. The $\alpha *$ 's denote the nondimensional flight times for the special case pointed out in [6] where the (intermediary) object and image positions coincide at the focal point of mirror $i$. for any velocity $v_{i}$. The signs for $\gamma_{i}$ may be chosen either as $\gamma_{i}=(-1)^{i-1}\left|\gamma_{i}\right|$ or as $\gamma_{i}=(-1)^{i}\left|\gamma_{i}\right|$. We may derive recursion and transfer relations in the form of linear fractions for the $\beta$ 's :

$$
\beta_{i}=\left(a_{i-1}^{i}+b_{i-1}^{i} \beta_{i-1}\right) /\left(c_{i-1}^{i}+d_{i-1}^{i} \beta_{i-1}\right)
$$
and

$$
\beta_{n}=\left(a+b \beta_{1}\right) /\left(c+d \beta_{1}\right) \text {. }
$$

We find $d_{i-1}^{l} \propto \gamma_{i}+\gamma_{i-1}$. Thus the relationships (11-12) display the special feature that for confocal systems (where the focal points of all mirrors coincide and thus $\left.\gamma_{i}=-\gamma_{i-1}\right) d$ and all the $d_{i-1}^{i}$ vanish. In this case the recursion and transfer relations become linear. This property is related to the special features of the confocal two-mirror microscope as well as of the general confocal $n$-mirror systems discussed below.

We can now formulate the conditions of achromatism. It follows from the symmetry properties of the
Taylor expansion coefficients for the total aberration $A$, that the only chromatic aberrations of the second and third order are given by $\partial^{2} A / \partial \phi \partial v, \partial^{2} A / \partial r \partial v$; and $\partial^{3} A / \partial \phi \partial v^{2}, \partial^{3} A / \partial r \partial v^{2}$, respectively, where the derivatives are taken at $\phi=0, r=0$, and for $v_{1}=V_{0}$. This type of chromatism may be designated as purely chromatic whereas a term like $\partial^{4} A /$ $\partial \phi^{3} \partial v$ which appears in fourth order may be classified as mixed geometrical-chromatic. The purely chromatic aberrations may be compensated in pairs by the choice of suitable mirror configurations satisfying the following conditions : a) $\delta_{n}^{\prime}=0$ and $M^{\prime}=0$, for compensation of the second-order terms ; b) $\delta_{n}^{\prime}=0, M^{\prime}=0$, and $\delta_{n}^{\prime \prime}=0, M^{\prime \prime}=0$ for elimination of the second and third order terms. Here and in the following the derivatives with respect to $\gamma$ are indicated by primes, and in the final expressions they are understood to be taken at $\gamma=1$, i.e., at the design velocity $v_{1}=V_{0}$. Chromatism beyond the third order is complicated by the presence of the mixed terms. It is not included in the present analysis. Numerically, it is negligible in the existing confocal two-mirror system, but in the highly corrected four-mirror system discussed below these residual aberrations become significant.

We may formulate the conditions of achromatism up to second or third order in various ways, using the transfer relations (7), (8), and/or (12). For instance, stationarity of image (and object) position up to second order $\left(\delta_{n}^{\prime}=0\right)$ implies $\tau_{n}^{\prime}=\tau_{n}^{-1}$ and $\tau_{1}^{\prime}=$ $\tau_{1}^{-1}$. Carrying the analysis up to the third order $\left(\delta_{\ell}^{\prime}=0\right.$ and $\left.\delta_{\ell}^{\prime \prime}=0\right)$ we have $\tau_{\ell}^{\prime \prime}-\tau_{\ell}^{-1}+\tau_{\ell}^{-3}=0$ (for $\ell=1$ and $\ell=n$ ). For the same conditions of longitudinal stationarity (in position) similar relations may be set up for the $\beta$ 's and their derivatives, and they may be used in conjunction with (12). For the present purpose it turns out to be sufficient to base the analysis on equations (8) in the form

and

$$
\begin{aligned}
& F_{1}(\gamma)=M_{1}^{-1}+X_{1} \tau_{1} \quad\left(=M^{-1}\right) \\
& F_{n}(\gamma)=M_{n}+X_{n} \tau_{n} \quad(=M)
\end{aligned}
$$

in combination with (7). Second-order stationarity of image/object position is ensured by the condition

$$
M F_{1}^{\prime}+M^{-1} F_{n}^{\prime}=0 \text {. }
$$


This implies complete absence of second-order chromatism for systems satisfying the conditions $F_{\ell}^{\prime}=0$ (for $\ell=1$ and $\ell=n$ ).

Similarly, the additional condition $M F_{1}^{\prime \prime}+$ $M^{-1} F_{n}^{\prime \prime}=0$ ensures third-order longitudinal stationarity (i.e., $\delta_{\ell}^{\prime \prime}=0$ ). Hence, a system is free of chromatism up to third order if $F_{\ell}^{\prime}=0$ and $F_{\ell}^{\prime \prime}=0$ (for $\ell=1$ and $\ell=n$ ).

These conditions of achromatism may be written explicitly in the form

$$
\begin{aligned}
F_{1}^{\prime}= & \left(M_{1}^{-1}\right)^{\prime}+X_{1}^{\prime} \tau_{1}+X_{1} \tau_{1}^{-1}=0, \\
F_{n}^{\prime}= & M_{n}^{\prime}+X_{n}^{\prime} \tau_{n}+X_{n} \tau_{n}^{-1}=0, \\
F_{1}^{\prime \prime}= & \left(M_{1}^{-1}\right)^{\prime \prime}+X_{1}^{\prime \prime} \tau_{1}+2 X_{1}^{\prime} \tau_{1}^{-1}+ \\
& +\left(X_{1} / \tau_{1}\right)\left(1-\tau_{1}^{-2}\right)=0 \\
F_{n}^{\prime \prime}= & M_{n}^{\prime \prime}+X_{n}^{\prime \prime} \tau_{n}+2 X_{n}^{\prime} \tau_{n}^{-1}+ \\
& +\left(X_{n} / \tau_{n}\right)\left(1-\tau_{n}^{-2}\right)=0 .
\end{aligned}
$$

In calculating the derivatives of $M_{\ell}$ and $X_{\ell}$ from the recursion relation (4) with use of (6) it is convenient to note the following relations : $\xi_{\nu}^{\prime}=\gamma / \xi_{\nu} \rightarrow \xi_{\nu}^{-1}$ (for $\gamma \rightarrow 1$ ), $\xi_{\nu}^{\prime \prime}=2 s_{\nu} / \xi_{\nu}^{3}$; similarly $\gamma_{i}^{\prime}=\gamma / \gamma_{i} \rightarrow$ $\gamma_{i}^{-1}$ and $\gamma_{i}^{\prime \prime}=\left(2 s_{i}+\rho_{i}\right) / \gamma_{i}^{3}$.

Confocal systems exhibit the interesting property that the lateral and longitudinal chromatism vanishes in pairs. I.e., condition $F_{1}^{\prime}=0$ (which may be used to determine $\tau_{1}$ from the quadratic equation (13)) implies $F_{n}^{\prime}=0$, while $F_{\ell}^{\prime}=0(\ell=1, n)$ and $F_{1}^{\prime \prime}=0$ imply that also $F_{n}^{\prime \prime}=0$. A proof of this property is sketched in the appendix.

As an example we consider the confocal twomirror system and find as the solutions of (13) and (14) (ensuring achromatism of second order)

and

$$
\left.\begin{array}{l}
\tau_{1}^{-1}=1-\omega(\mp) u \\
\tau_{2}^{-1}=-1+\omega(\mp) u,
\end{array}\right\}
$$

where

$u=\left\{\omega\left[\omega(\omega-1)\left(1+\rho_{1}\right)+\rho_{1}\right] /\right.$

$$
\left.\left[\omega\left(1+\rho_{1}\right)-1\right]\right\}^{1 / 2}
$$

and

$$
\omega=\xi_{1} / \xi_{2}=\xi_{2}^{-1}
$$

for our choice of the reference level $s=0$ at mirror 1 , which implies $s_{1}=0$ and $\xi_{1}=\gamma \rightarrow 1$ for $\gamma \rightarrow 1\left(^{2}\right)$. As a consequence of confocality $\omega$ is related to the mirror radii in the form $\omega^{2}=$ $\left(1+\rho_{1}-\rho_{2}\right)^{-1}$.

$\left({ }^{2}\right)$ In paper [6] we had used mirror 2 as the reference level. Alternatively, if we restrict ourselves to the discussion of confocal systems, we might choose with advantage the common focal point as the origin. In the latter system of coordinates the following simple relations hold : $s_{i}=-\rho_{i} / 2$ and $\gamma_{i}^{2}=\gamma^{2}$ for all $i \leqq n$.
Inspection of (17) reveals that the only physically meaningful solution, which corresponds to real, not virtual, object and image points, is obtained for $\omega<0$ (i.e., reversal of flight direction between the mirrors) and for the choice of the negative signs in (17). Formally, object and image (both virtual) exactly change places in the second algebraic solutions. This does not correspond to a mere time reversal since the sequence of mirror reflections remains unchanged. All these results are consistent with, and they complement, the previous analysis in reference [6].

It seems interesting to note that an $n$-mirror system of the type discussed can physically consist of $n^{*}<n$ mirrors one or more of which is-are hit more than once. Since physically coincident mirrors are necessarily confocal we can, e.g., realize an achromatic system (i.e., full achromatism up to second order, which is not possible for the simple singlemirror system) by use of only one concave mirror from which the particle is reflected twice. This is the special case $R_{2}=R_{1}(<0)$ of the confocal two-mirror system discussed above, and it displays similar topological features. In this special case both solutions of (13-14) are physically possible, and only in this instance do they correspond to time-reversed trajectories. In contrast with the existing microscope set-up where $R_{2}>0$ the intermediary image is real for $R_{2}<0$. For large magnifications it is formed near the object. Consequently, the object support would block a substantial fraction of trajectories. This feature is even more pronounced for the degenerate confocal $n$-mirror system with $n>2$, where the particles bounce $n$ times on the same mirror before forming an achromatic image. Higher-than-secondorder achromatism cannot be achieved in this scheme, and for given $R_{i}=R_{1}$ (for $i=2, \ldots, n$ ) the focussing conditions are never the same for different $n$. Thus, for achromatic setting the intermediary images are always chromatic. These properties are a direct consequence of the quadratic nature of equations (13-14). Generally, only one solution $\tau_{1}$ exists for (13-14) in conjunction with the transfer relation (7). In conditions of confocality (13) and (14) are linearly dependent but only one of the two algebraic solutions corresponds to a physical solution.

We add one further practical implication of the intrinsic achromatism of confocal systems. In the confocal two-mirror microscope more than one reflection can take place successively on mirror 2 , and the system so obtained is again confocal. For any design setting of the microscope, however, the higher-order images are out of focus and very weak because the magnification strongly increases with the number of reflections on mirror 2 . We may, however, consider imaging systems with multiple reflections and multiple reversals of beam trajec- 
tories for sensitive searches for beam deflections due to small lateral forces.

From the foregoing analysis we could expect to find systems with more than two distinct mirrors which are fully achromatic up to third order, i.e., which satisfy equations (13-16). A systematic numerical scan of possible geometries for up to five confocal mirrors over a wide range of parameters has, however, revealed no feasible system with real object and image. This situation can apparently not be remedied by a departure from confocality under the constraints imposed by equations (13-16). However, the problem is relaxed if we are content with partial compensation of third-order chromatism. In section 5 a feasible scheme of a four-mirror microscope will be presented where second-order chromatism is fully corrected along with third-order chromatism of image position, and where the resolution due to the residual total aberration (including the chromatic, geometric and the mixed types) is reduced to a few $\mu \mathrm{m}$.

\section{Mirror shapes.}

We consider again the special one-mirror system [6] where the object and image positions coincide at the focal point, for any velocity. The image is thus free of longitudinal chromatic aberrations $\left(\propto \partial^{m+1} A\right.$ ) $\partial \phi \partial v^{m}$ ) to all orders $m$, while transversal chromatism occurs in all orders. Object and image are both real for $R<0$ (focal point above the mirror), while one of them is virtual for $R>0$. It may be shown from the general properties of the flight parabola that the longitudinal achromatism of this system is not restricted to paraxial rays if we choose a parabolic mirror shape. A particle emanating from the focal point in any direction and at any given speed is reflected back into its origin by the mirror which we assume to be of infinite extent. The image is thus completely free both of longitudinal chromatism and of spherical aberration. I.e., $\partial^{m} A / \partial \phi^{m}=0$ for $m=3,5,7 \ldots$ In view of these unique properties we can consider this system as the generalisation to particle optics in a homogeneous force field $\mathbf{g}$, of straight-ray imaging by a parabolic mirror. (For $g=0$ the focal rays will, of course, not actually return to the focal point from infinity, but for arbitrarily small $g$ they would.) The analogy with the straight-ray case is also evidenced by the unique property of constant flight-time between the coincident object and image, irrespective of initial flight direction. This is a special case of Fermat's principle, and again reminiscent of light optics, since Fermat's principle of stationary phase (which holds for any imaging system) does not normally imply stationary flight times in massive particle optics [16].

The remarkable properties of this system (the «neutron fountain ») constitute the basis for the development of high-speed, highly magnifying optical systems for neutrons. In practical cases the rise height $\left(V_{0}^{2} / 2 g\right.$ as measured from the mirror level) is always large compared with the focal lengths, thus $|\rho| \ll 1$. Consequently, the special one-mirror system is highly magnifying or reducing, depending on whether the initial ray is directed to, or away from, the mirror. This can be seen from the expression $\mu^{ \pm 1}=-(1+\sqrt{1+\rho})^{2} / \rho \cong-4 / \rho$ for the magnification. In practical cases we need two or more mirrors for chromatic compensation. The (intermediary) object/image positions of none of these mirrors will exactly correspond to the special configuration. However, they can be made to approach this case, and the relevant criterion is the magnification (or reduction). Thus, the primary mirror(s) of large aperture should be strongly magnifying (or reducing). A small deviation from the prototype system can then be taken into account by a slight departure from the parabolic mirror shape without encountering a significant loss in angular aperture and longitudinal achromatism.

The existing confocal two-mirror microscope (Fig. 1) is an example for this strategy. Both the object and the intermediary image lie close to the focal point of mirror 1 for all settings. Therefore the parabolic shape is a good choice. We could further reduce the spherical aberrations to insignificant values if instead of the parabola we chose a slight elliptical deformation with eccentricity $\varepsilon \cong 1-10^{-3}$ (where $\varepsilon=1$ corresponds to the parabolic limit and the value of $\varepsilon$ can be optimized analytically for any desired setting). The shape of the second mirror is irrelevant because of the small beam divergency $\left(\simeq 2 \phi / \mu_{1}\right)$ incident on it.

In the confocal two-mirror system we cannot correct coma and similar lateral aberrations related to the object size $r_{\max }$. This feature is again clear from the prototype single-mirror case where the lateral aberrations play the dominant role. We shall now discuss a four-mirror system where an appropriate choice of mirror shapes allows us to eliminate coma along with spherical aberration and to carry achromatism beyond the lowest order.

\section{A four-mirror system.}

The scheme outlined in figure 4 consists essentially of two stages which are based on the two possible achromatic two-mirror systems : the Frank optics [9] used as the initial stage (mirrors 1 and 2) and the confocal second stage (mirrors 3 and 4). In this configuration it is possible to improve on the overall characteristics by a relaxation of the geometrical constraints for the two constituent subsystems. Thus, neither are mirrors 3 and 4 strictly confocal nor do mirrors 1 and 2 satisfy the two-mirror con- 


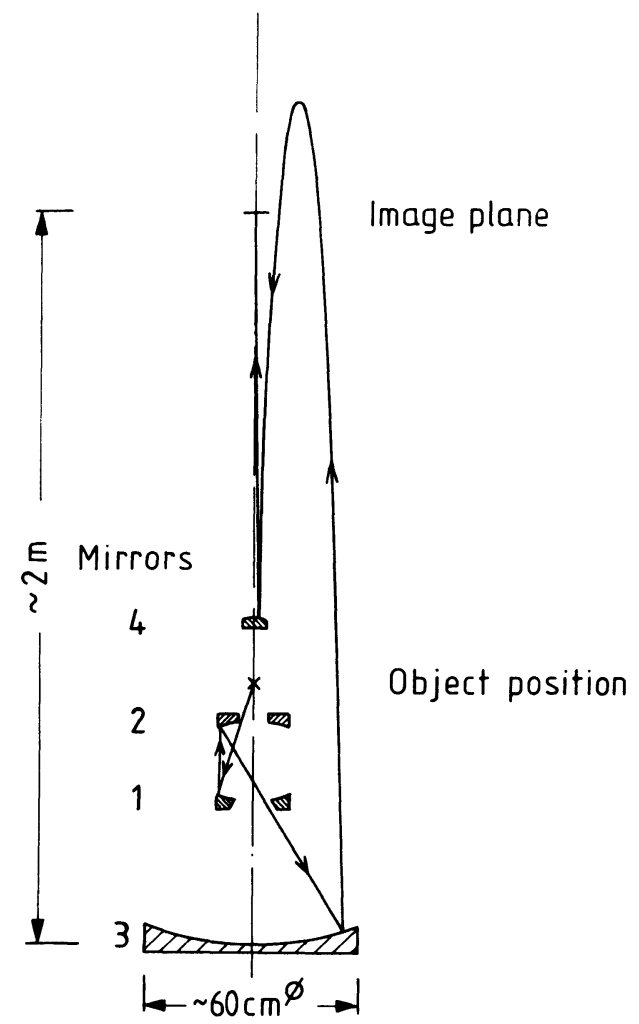

Fig. 4. - Scheme of a high-speed, high-magnification, achromatic and aplanatic four mirror neutron microscope optics. It consists essentially of two double-mirror systems: The Frank optics as the initial stage and the confocal system used as the highly magnifying second stage.

dition of achromatism ( $\left.{ }^{3}\right), \rho_{2} \xi_{2}-\rho_{1}=2\left(1-\xi_{2}^{3}\right)$. The deviations were determined by the postulate to compensate the third-order longitudinal aberration $\left(\propto \partial^{3} A / \partial \phi \partial v^{2}\right)$ in addition to both second-order terms. The intermediary image between the two double-mirror subsystems then is no longer achromatic but it is still formed very near the focal point of mirror 3. While it is not possible in this scheme to compensate both third-order chromatic aberrations, it offers enough flexibility to design an aplanatic neutron optics with small residual total aberration.

As an example we consider a system with magnification $M=109.67$, characterized by the following nondimensional parameters : $\rho_{1}=-0.15, \rho_{2}=$ $0.095, \quad \rho_{3}=-0.23, \quad \rho_{4}=0.1 ; \quad s_{1}=0, \quad s_{2}=$ $-0.054176, \quad s_{3}=0.106, \quad s_{4}=-0.12961 ; \quad \mu_{1}=$ $7.8583, \mu_{2}=-0.076339$ (thus the first stage is reducing by the factor $\left.\mu_{1} \mu_{2}=-0.5999\right)$ and

$\left({ }^{3}\right)$ For sufficiently high UCN velocities this exact condition for the Frank optics reduces to the relation $s_{2}=\left(\rho_{1}-\rho_{2}\right) / 6$ for the mirror separation given in reference [9]. We use here the nomenclature and the sign conventions of the present analysis and set again $s_{1}=0$, $\xi_{1}=1$. $\mu_{3}=18.2791, \mu_{4}=-10.001$ (which corresponds to a magnification of $\mu_{3} \mu_{4}=-182.82$ for the second stage). For a design velocity of $V_{0}=6.1893 \mathrm{~m} / \mathrm{s}$ we obtain the following real dimensions: $R_{1}=$ $-0.58592 \mathrm{~m}, R_{2}=0.37108 \mathrm{~m}, R_{3}=-0.89841 \mathrm{~m}$, $R_{4}=0.39061 \mathrm{~m} ; S_{1}=0, S_{2}=-0.21162 \mathrm{~m}, S_{3}=$ $0.41405 \mathrm{~m}, S_{4}=-0.50626 \mathrm{~m}$. The optimum object position lies $0.31628 \mathrm{~m}$ above mirror 1 , and the image is formed $1.22398 \mathrm{~m}$ above mirror 4 where the design neutron velocity amounts to $2.1 \mathrm{~m} / \mathrm{s}$.

Similarly as in the Schwarzschild-type neutron optics for very cold neutrons [7] the configuration of figure 4 may be made aplanatic by a considerable deviation of the shapes of mirrors 1 and 2 from a parabola. The present design is, however, not only aplanatic (as in Ref. [7]) but also achromatic. We have optimized the system with respect to minimum mean squared total aberration (as approximately by the condition of minimum quadratic sum of the differential aberrations up to 9th order) over an image field width of $6 \mathrm{~cm}$ in diameter, a primary aperture of $2 \times 0.36 \mathrm{rad}$ and a velocity range of $\pm 0.27 \mathrm{~m} / \mathrm{s}$ about $V_{0}$. In this way the following mirror shapes were obtained :

Mirror 1 : elliptical with eccentricity $\varepsilon_{1}=0.463$; Mirror 2: hyperbolic with $\varepsilon_{2}=1.363$; Mirror 3 (of diameter $60 \mathrm{~cm}$ ) : elliptical (nearly parabolic) with $\varepsilon_{3}=0.9844$ and a fourth-order (wide-angle) correction to the ellipsoid of the form $\left(z_{3}-S_{\mathrm{c} 3}\right)^{2}=$ $\chi^{2} R_{3}^{2}-\chi\left(x^{2}+y^{2}\right)+\chi \psi\left(x^{2}+y^{2}\right)^{2} / R_{3}^{2}$, where $\chi=$ $\left(1-\varepsilon_{3}^{2}\right)^{-1} \cdot S_{\mathrm{c} 3}=S_{3}+R_{3} \chi$ denotes the centre position of the ellipsoid, and the best fitted value for the correction is given by $\psi=-0.0371$; Mirror 4 : spherical $\left(\varepsilon_{4}=0\right)$. For this design the aberration limit to the resolution amounts to a few $\mu \mathrm{m}$.

As in the confocal double-mirror set-up the geometry may be adjusted to a higher magnification most easily by the choice of a smaller radius of curvature for the fourth mirror, accompanied by an appropriate adjustment of mirror separations and for optimum performance - of mirror shapes.

\section{Conclusions.}

Using an achromatic, confocal system of two mirrors for ultracold neutron imaging we have obtained sharp neutron images of an object slit. A resolution of $10 \mu \mathrm{m}$ was observed at magnifications up to 240 . We have shown that a further improvement in these characteristics can be expected for systems with more than two mirrors. A special four-mirror arrangement has been proposed which would reduce the aberration limit to the resolution to a few $\mu \mathrm{m}$.

We expect that neutron microscopy may be applied in the future to study surface magnetism and the spatial distribution of hydrogenous structures on biological specimens. 


\section{Appendix.}

A proof of the special properties of confocal systems may be based on their transfer properties. It follows from (5) and (9) that for $n$ confocal mirrors (where $\gamma_{i}^{2}=\gamma_{1}^{2}$ for all $i$ )

$$
C=(-1)^{n} B \text { and } D=(-1)^{n} E / \gamma_{1}^{2},
$$

hence, with use of (10) :

$\tau_{n}=(-1)^{n}\left(\gamma_{1}^{2} M_{1} X_{1}+\tau_{1}\right) /\left(1+M_{1} X_{1} \tau_{1}\right)$,

and

$$
M_{n}=M_{1}^{-1}, \quad X_{n}=-(-1)^{n} X_{1} .
$$

In the last expressions we have employed the relation

$$
M_{1}^{-2}-\gamma_{1}^{2} X_{1}^{2}=1 .
$$

To show this identity we consider the special case where object, image and all the intermediary images coincide at the common focal point, i.e. $\tau_{1}^{* 2}=$ $\tau_{i j}^{* 2}=\tau_{n}^{* 2}=\gamma_{1}^{2}$. According to equation (8a) the magnification $M^{*}$ is given by

$$
M_{ \pm}^{*-1}=M_{1}^{-1} \pm \tau_{1}^{*} X_{1}
$$

The \pm signs correspond to the two adjoint possibilities where the initial ray from the object is directed towards the first mirror or away from it, respectively. The system considered consists of a sequence of the special one-mirror systems with coincident object and image at the common focal point. For each subsystem $i$ the respective partial magnifications are related in the form

$$
\mu_{i+}^{*-1}=\mu_{i-}^{*} .
$$

Hence the total magnifications

$$
\begin{aligned}
M_{+}^{*-1}=\mu_{1+}^{*-1} \mu_{2+}^{*-1} & \ldots \mu_{n+}^{*-1}= \\
& =\mu_{1-}^{*} \mu_{2}^{*} \ldots \mu_{n-}^{*}=M_{-}^{*}
\end{aligned}
$$

are also reciprocal. Therefore

$M_{+}^{*-1} M_{-}^{*-1}=\left(M_{1}^{-1}+\tau_{1}^{*} X_{1}\right)\left(M_{1}^{-1}-\tau_{1}^{*} X_{1}\right)=1$,

which proves (A.3).

Combining (A.3) and its derivatives at $\gamma=1$, as well as (A.2) and (7) with equation (13) (for the second-order aberrations, or with (13) and (15) for the second and third order aberrations) we can verify that equation (14) (or (14) and (16), respectively) is/are satisfied at the same time. Hence, for confocal systems the longitudinal and transversal chromatism of second and third order vanishes in pairs.

We can go one step further and show that the pure chromatic aberrations of any order $m \geqq 2$ vanish in pairs. Similarly as for the $\beta$ 's (cf. Sect. 3) we relate the general problem of imaging by $n$ confocal mirrors to the special case of focal position for the object and its image where the velocities are related by $\tau_{n}^{*}=(-1)^{n} \tau_{1}^{*}$. The velocities change sign between successive mirrors because the intervening flight trajectory always passes an apex. Using this property we may derive from (A.1-2) the following relations for the relative velocities $\tau_{\ell} \mp \tau_{\ell}^{*}$ (for $\ell=1$ or $n)$ :

$$
\begin{aligned}
X_{n}=\left(M_{1}^{-1} \pm \tau_{1}^{*} X_{1}\right) / & \left(\tau_{1} \mp \tau_{1}^{*}\right)- \\
& -\left(M_{n} \pm \tau_{n}^{*} X_{n}\right) /\left(\tau_{n} \mp \tau_{n}^{*}\right),
\end{aligned}
$$

or, by subtraction of these identities,

$$
Q=\gamma_{1} F_{1} / \delta_{f 1}-\gamma_{n} F_{n} / \delta_{f n}=0 .
$$

Here $F_{\ell}$ is defined by equations $(8 \mathrm{a}, \mathrm{b})$ and $\delta_{f \ell}=\left(\tau_{\ell}^{2}-\gamma_{1}^{2}\right) / 2$ denotes the nondimensional object distance (for $\ell=1$ ) and image distance (for $\ell=n$ ) from the common focal point. Combining (A.5) with the relation

$$
M F_{1}-M^{-1} F_{n}=0
$$

which follows from $(8 a, b)$, we obtain the following key property of confocal systems :

$$
M^{2}=\delta_{f n} / \delta_{f 1}=d_{f n} / d_{f 1},
$$

where $d_{f \ell}$ denotes the real object/image distance below the focal point. Relation (A.7) holds for any velocity $V_{0}$ and for any number $n$ of confocal mirrors. For a single mirror $i$ it may be written in the form

$$
\mu_{i}^{2}=\delta_{f i 2} / \delta_{f i 1}=d_{f i 2} / d_{f i 1},
$$

where the third index again refers to (intermediary) object $(j=1)$ and image $(j=2)$, respectively. Equation (A.8) is consistent with the imaging equation (2). The connection (A.7) between object and final image may also be demonstrated by repeated application of (A.8) since in conditions of confocality $\delta_{f(i+1) 1}=\delta_{f i 2}$. Relations analogous to (A.7) hold also between arbitrary intermediary images and objects. Equation (A.8) is well known in light optics which may be considered as the straightray limit $\left(V_{0} \rightarrow \infty\right)$.

It follows immediately from (A.7) that for confocal systems all the higher-order derivatives of $M$ and $\delta_{f n}$, and thus also the pure longitudinal and transversal aberrations, can only vanish in pairs, provided that all the lower-order derivatives are zero. Choosing suitable geometries for $n$ confocal mirrors we can, in principle, eliminate the pure chromatic aberrations up to the order $m=n-$ although it is not clear whether physically possible systems with real object and image exist beyond $n=2$. 


\section{Acknowledgments.}

This work has been funded by the German Federal Minister for Research and Technology (BMFT) under the contract number 03-St1 TUM 4. We are grateful to H. Nagel and F.-X. Schreiber for excellent technical help, to the Department of Reactor Operation at the Institut Laue Langevin for the good collaboration, and to the ILL direction for their support. Ph. Ledebt, F. Thurel and R. Klesse whave contributed to the solution of computing problems. One of us (T.E.) is very grateful for the hospitality extended to him during his stay at ILL. Last not least we thank Mrs. G. Creighton for typing the manuscript.

\section{References}

[1] Frank, I. M., Priroda 9 (1972) 24.

[2] Steyerl, A. and Schütz, G., Appl. Phys. 17 (1978) 45.

[3] Schütz, G., Steyerl, A. and Mampe, W., Phys. Rev. Lett. 44 (1980) 1400.

[4] HerrmanN, P., « Ein Neutronenmikroskop für ultrakalte Neutronen : Entwicklung und erste Messungen », Dipl. thesis, Technische Universität München, 1982.

[5] Arzumanov, S. S., Masalovich, S. V., Strepetov, A. N. and Frank, A. I., Pis'ma Zh. Eksp. Teor. Fiz. 39 (1984) 486 [JETP Lett. 49 (1984) 590].

[6] Herrmann, P., Steinhauser, K.-A., Gähler, R., Steyerl, A. and MAMPe, W., Phys. Rev. Lett. 54 (1985) 1969.

[7] Steyerl, A. and MAlik, S. S., in C. G. Shull Symposium (Cambridge, U.S.A., Sept. 1985), Physica 137B (1986) 270 («Frontiers of Neutron Scattering » Eds R. I. Birgenau, D. E. Moncton and A. Zeilinger).

[8] Strepetov, A. N. and. Frank, A. I., Pis'ma Zh. Eksp. Teor. Fiz. 12 (1986) 71.

[9] Arzumanov, S. S., Strepetov, A. N., Masalovich, S. V. and Frank, A. I., Pis'ma Zh. Eksp. Teor. Fiz. 44 (1986) 213.
[10] Frank, A. I., V International School on Neutron Physics, Alushta, Oct. 1986, ed. by Jaint Institute for Nuclear Research, Dubna, D3, 4, 17-86747 (1987).

[11] Steyerl, A., Nagel, H., Schreiber, F.-X., Steinhauser, K.-A., Gähler, R., Gläser, W., Ageron, P., Astruc, J.-M., Drexel, W., Gervais, R. and MAMPE, W., Phys. Lett. A 116 (1986) 347

[12] Skachkova, O.S. and Frank, A. I., Pis'ma $Z h$. Eksp. Teor. Fiz. 33 (1981) 214 [JETP Lett. 33 (1981) 203].

[13] Strepetov, A. N. and Frank, A. I., Zh. Tekhn. Fiz. 56 (1986) 1775.

[14] Ageron, P., Hetzelt, M., Mampe, W., PendleBURY, J. M., RobSON, J. M. and SMITH, K., in Proceedings of the International Symposium on Neutron Inelastic Scattering, Vienna, 1977 (International Atomic Energy Agency, Vienna, 1978), Vol. 1, p. 53.

[15] Golub, R., Jewell, C., Ageron, P., Mampe, W., Heckel, B., Kilvington, I., Z. Physik B 51 (1983) 187.

[16] Steyerl, A., J. Phys. Colloq. France 45 (1984) C3255. 